\title{
Modeling and Containment of Search Worms Targeting Web Applications
}

\author{
Jingyu Hua*, Kouichi Sakurai \\ Information Technolgy \& Security Lab \\ Kyushu Univ.
}

Speaker: Jingyu Hua Email: huajingyu@gmail.com

* He is partly supported by the Grant of Graduate school of ISEE of Kyushu University for Supporting Students' Overseas Traveling and the China Governmental Scholarship. 


\section{Outline}

$\square$ Background: What are Search Worms?

$\square$ Motivation: Modeling \& Containing

$\square$ Modeling of Search Worms

$\square$ Containment of Search Worms

$\square$ Conclusion

$\square$ Future Work 


\section{Background-Google Hacking}

Google is a great tool for Hackers!!!

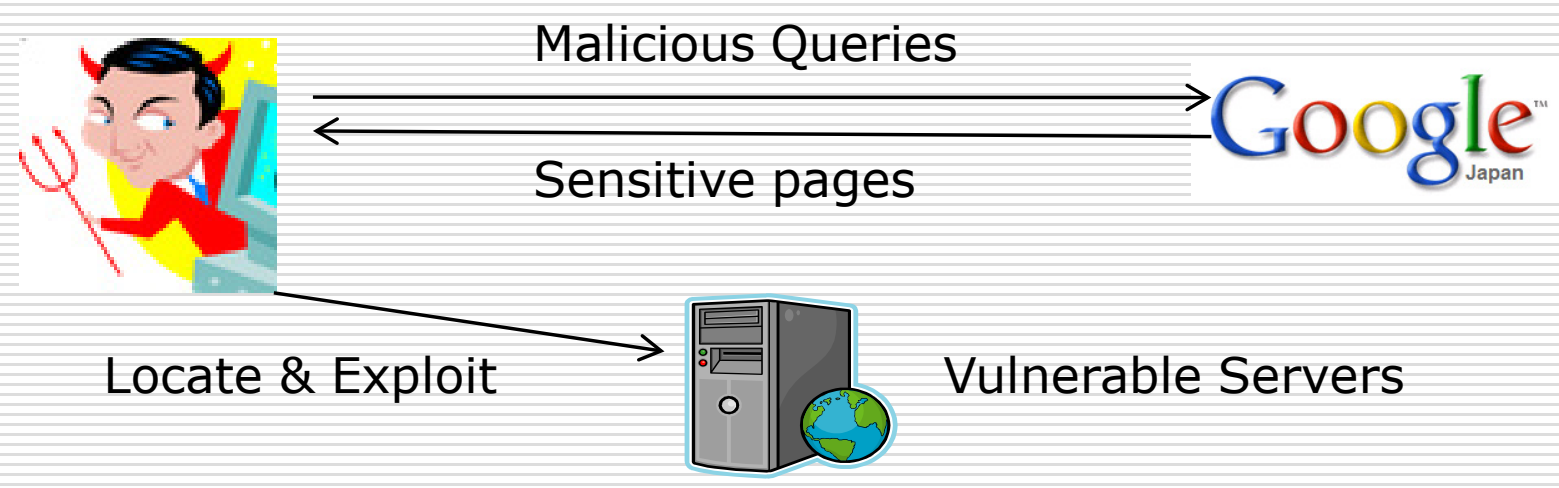

Google filetype:inc intext:mysql_connect ○所有网页 $\bigcirc$ 中文网页 $\bigcirc$ 简体中文网页

苅 $甲$ 打井百宝箱...

functions.inc - Index of / - [ [ i

$<$ ?php function ListRedTree(\$Start) \{ \$Node=array(); \$DBLink=mysql_connect("localhost",

"guest"); mysql_select_db("menutest",\$DBLink); \$Query="SELECT ID, ...

hclements.com/Menu/functions.inc - 网页快照 $-\ominus$ 园平

Eigenpages: dangerous pages that can disclose server vulnerabilities

Google 搜索 高级

ㅁ $3 / 22$

2010/7/9 DIMVA'10 


\section{Background-Search Worm}

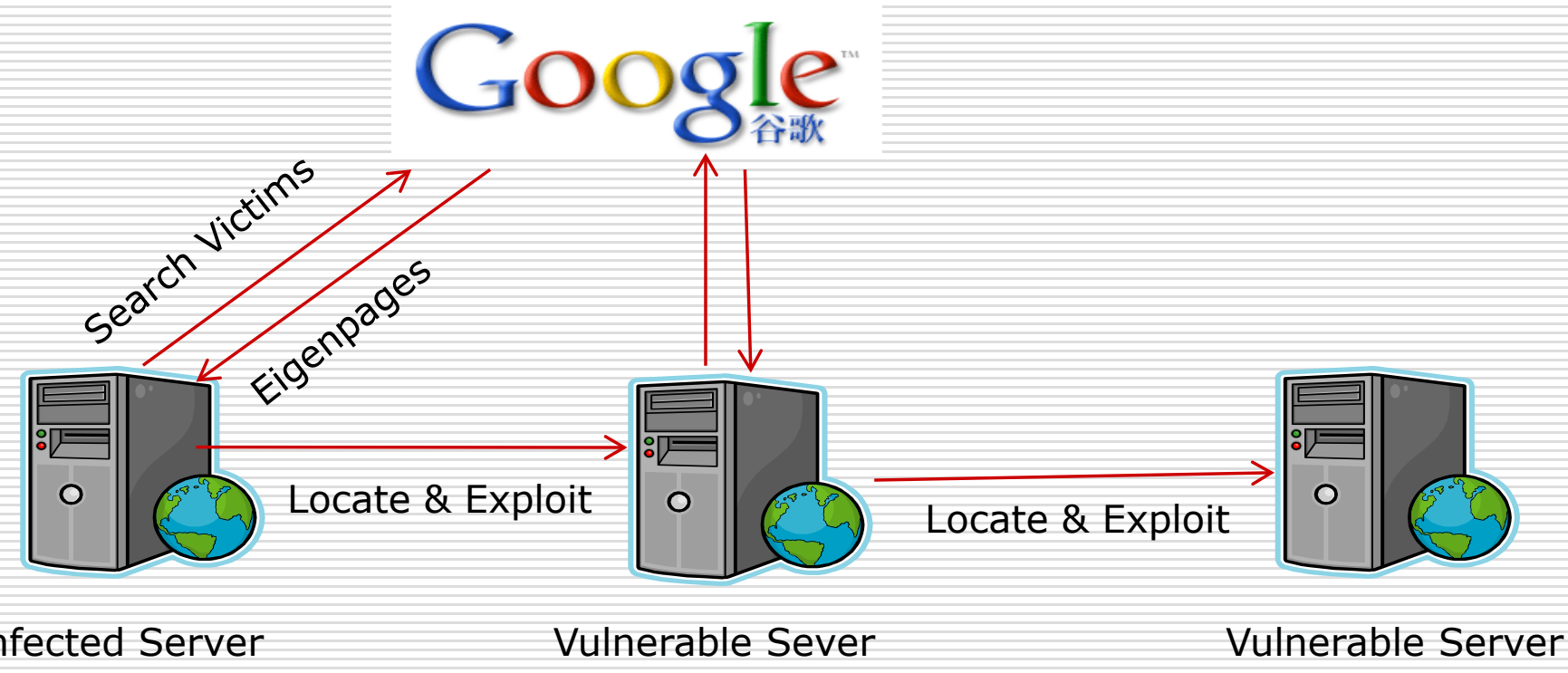

Search Worm: a worm uses search engines to locate targets

Example:

Santy: targeting phpBB bulletin system.

Google Dork: allinurl: viewtopic.php

Released on $12 / 20 / 2004$, infected 40,000 servers in 2 days 


\section{Motivation}

$\square$ Search Worms are dangerous! So, It deservers our research.

$\square$ Two common problems in the field of worm studying:

- How to model the propagation of such worms ?

$(\mathrm{I}(\mathrm{t})=$ ?)

$\square$ Study the spreading characteristics

$\square$ Study the effects of containment strategies

- How to contain the propagation of such worms? 
Topic 1

Modeling of Search Worms 


\section{Modeling of the Search Worms A virtual search worm}

$\square \quad$ Vulnerable severs leak eigenpages containing specific keywords to search engine.

$N$ : num of severs containing the eigenpages (Suspicious severs)

V: num of really exploitable severs (Vulnerable servers)

$\square$ Propagation steps of infected severs:

1. Search "special-keywords and random-keywords" in a search engine $\rightarrow m$ search results

2. Choose $\delta$ pages among the total $m$ search results to scan.

3. Once a server is infected, it begins this infection cycle, too. 
Modeling of the Search Worms

Effects of Eigenpage Distribution

$\square$ Obviously, severs contain more eigenpages are more likely to be exploited.

$\square$ Two attacker-favorable assumptions:

- $m$ search results are randomly selected from all the eigenpages on the web

- $\delta$ targets are randomly selected from the $m$ search results 


\section{Modeling of the Search Worms Effects of Eigenpage Distribution}

$\square$ U-Model: eigenpages are uniformly distributed on severs

The number of infected servers by the end of time tick $t$ :

$$
I(t)=I(t-1)+\left[\begin{array}{|c|c|c|}
{[V-I(t-1)]} & \left.1-\left(1-\frac{1}{N}\right)^{\delta I(t-1)}\right] & \begin{array}{l}
\text { Newly infected } \\
\text { severs during } \\
\text { the time tick } t
\end{array} \\
\begin{array}{c}
\text { Remained vulnerable } \\
\text { severs by the end of } \\
\text { the time tick } t-1
\end{array} & \begin{array}{l}
\text { The probability that } \\
\text { a specific server is } \\
\text { hit by a scan during } \\
\text { the time tick } t
\end{array}
\end{array}\right.
$$

$V$ is the total count of servers really suffering the vulnerabilities among the $\mathrm{N}$ severs containing eigenpages 


\section{Modeling of the Search Worms Effects of Eigenpage Distribution}

$\square$ PL-Model: eigenpages follow a power law distribution.

The probability that the number of eigenpages $p$ on a suspicious server is greater than $x$ is

$$
\begin{aligned}
& \operatorname{prob}(p>x)=\left(\frac{p_{\text {min }}}{x}\right)^{\sigma} \begin{array}{c}
\text { N suspicious } \\
\text { servers }
\end{array} \\
& \text { Divided into } k \text { groups } \\
& 10 \text { pages } 100 \text { pages } \\
& a_{i}=N \cdot\left[\operatorname{prob}\left(p>p_{i}\right)-\operatorname{prob}\left(p>p_{i+1}\right)\right]=N \cdot\left[\left(\frac{p_{m i n}}{p_{i}}\right)^{\sigma}-\left(\frac{p_{\text {min }}}{p_{i+1}}\right)^{\sigma}\right]
\end{aligned}
$$




\section{Modeling of the Search Worms Effects of Eigenpage Distribution}

$\square$ PL-Model:

$$
\begin{aligned}
& \text { PL-Model: } \\
& I(t, i)=I(t-1, i)+\left[V_{\imath}-I(t-1, i)\right]\left[1-\left(1-\frac{p_{i}}{P}\right)^{\delta I(t-1)}\right] \\
& I(t)=\sum_{i=1}^{k} I(t, i)
\end{aligned}
$$
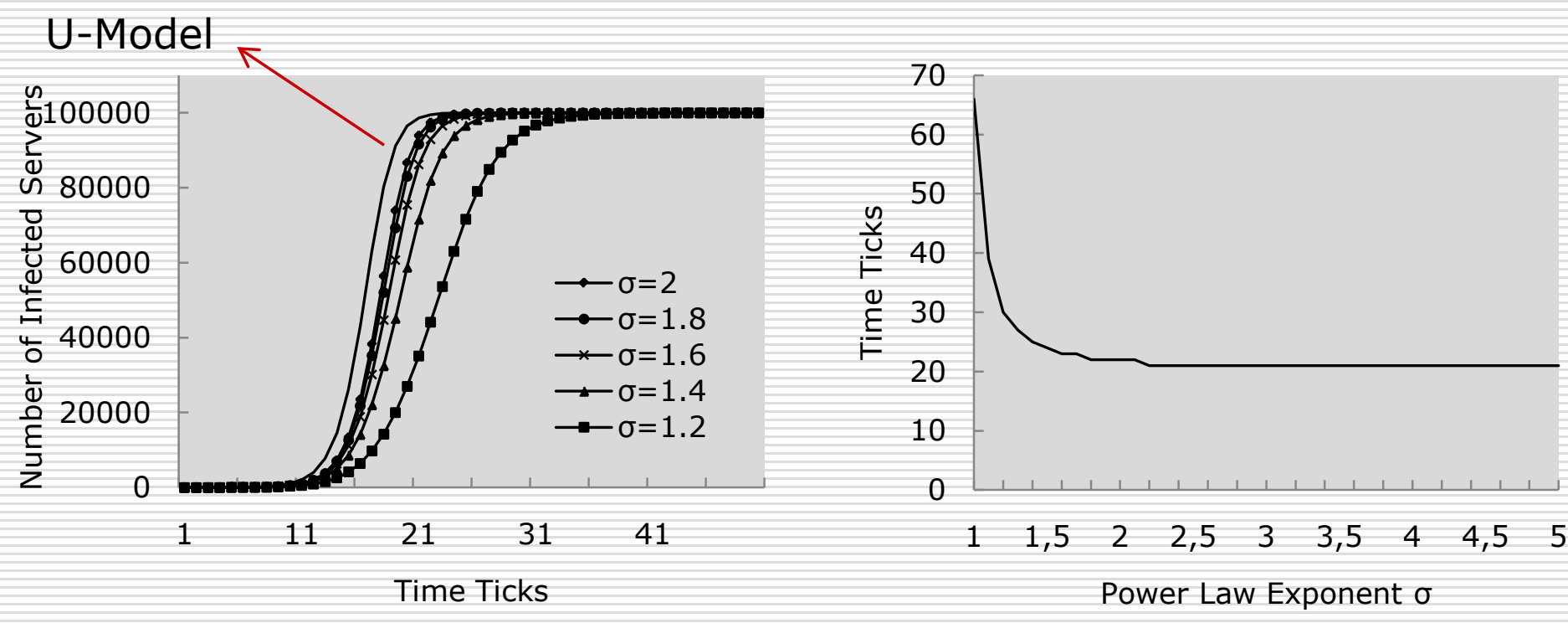

Time to infect $95 \%$ vulnerable severs for the PL-Model 
Modeling of the Search Worms

Effects of Eigenpage Distribution

$\square$ Proposition:

Among different distributions of eigenpages, the uniform distribution optimizes the performance of search worms.

We proved this conclusion by using the mean value inequality 


\section{Modeling of the Search Worms Effects of Page Ranking}

$\square$ Search results are ranked according to Keyword relevance, page importance....

$\square$ Pages on popular servers are more likely to appear in front $\rightarrow$ scan collisions.

$\square$ If the second attacker-favorable assumption is true, no scan collisions.

Google

However, the second assumption is impossible, the propagation of search worm will be affected by page ranking 


\section{Modeling of the Search Worms Effects of Page Ranking}

$\square$ Page importance:

- Page Ranking Value (0-10)

Power Law [Litvak 2007]

$$
\operatorname{prob}(P R=k)= \begin{cases}\left(\frac{1}{6^{k}}\right)^{\alpha}-\left(\frac{1}{6^{k+1}}\right)^{\alpha} & 0 \leq k<10 \\ \left(\frac{1}{6^{10}}\right)^{\alpha} & k=10\end{cases}
$$

- Site importance

We simply assume servers contain more pages are more important

[Litvak 2007] Litvak, N., Scheinhardt, W. R. W., Volkovich, Y.: In-degree and PageRank: Why do they follow similar power laws? Internet Math, Vol.4(2-3), pp.175-198 (2007) 


\section{Modeling of the Search Worms Effects of Page Ranking}

\section{$\square$ PR-Model:}

An infected server selects the $\delta$ tod-ranking

$$
\left\{\begin{array}{l}
I(t, i)=I(t-1, i)+\left[V_{i}-I(t-1, i)\right]\left[1-\left(1-\frac{1}{a_{i}}\right)^{\delta_{i} I(t-1)}\right] \\
I(t)=\sum_{i=1}^{k} I(t, i)
\end{array}\right.
$$

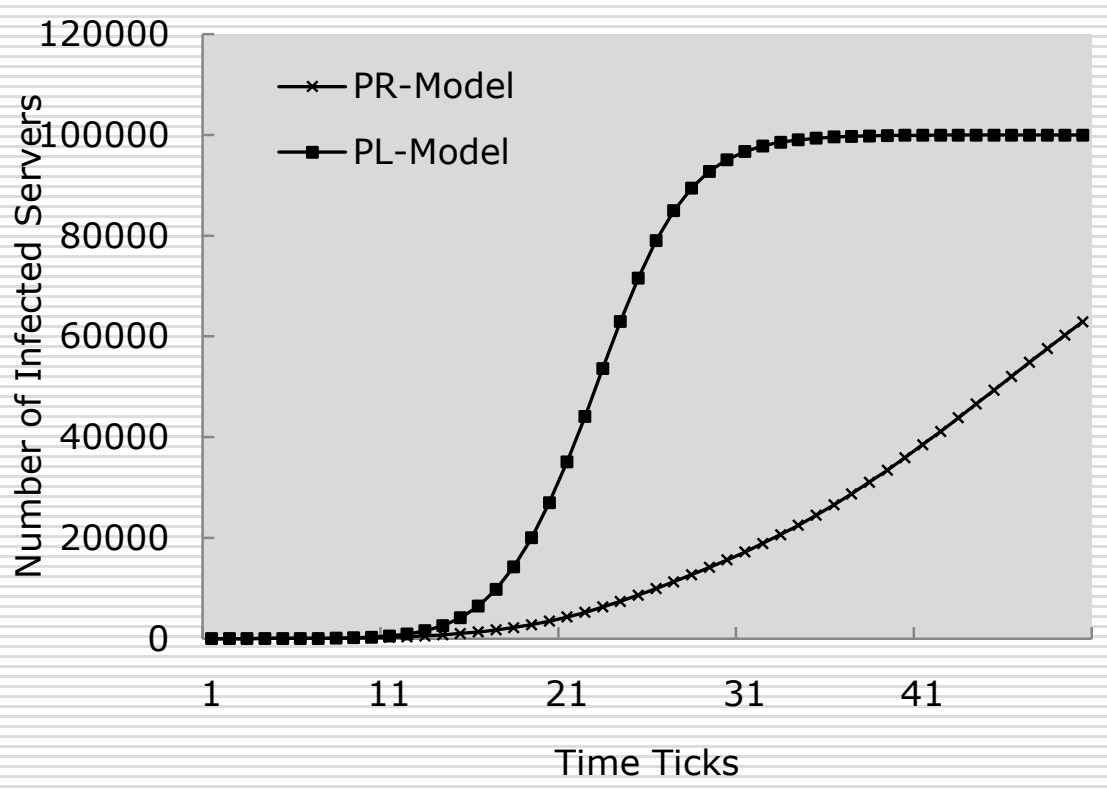

Page ranking slows down the spreading of the search worm 


\section{Topic 2:}

Containment of Search Worms 


\section{Containment of the Search Worm}

$\square$ The goal to model the search worm is to help developing an efficient containment system.

$\square$ We introduce a conceptual containment system based on honey-page insertion.

$\square$ We use our propagation models to analyze this system. 


\section{Containment of the Search Worm}

$\square$ Honey Page
Google "index of /etc/passwd"
Search Advanced Search

Web $\boxplus$ Show options...

Results $1-10$ of about 2

\section{Index of /etc/passwd}

Index of /etc/passwd. Name Last modified Size Description. [DIR] Parent Directory 31-Jul-

2003 12:36 - [TXT] AT-admin.cgi 31-Jul-2003 12:55 2k [TXT] ...

gray-world.net/etc/passwd/ - Similar - $\risingdotseq$ 还

This is a honey page pointing to a honey pot 


\section{Containment of the Search Worm}

$\square$ Containment based on honey-page insertion:

Search engine randomly inserts honey-pages into search results for any query.

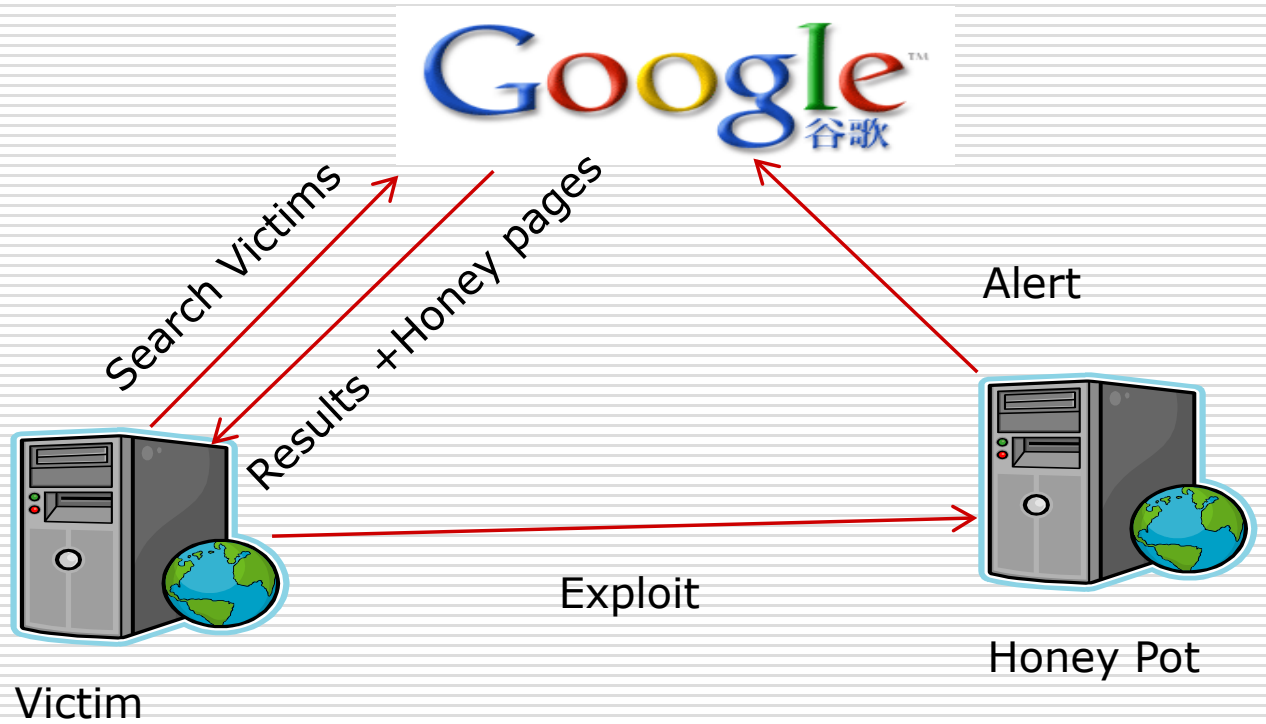

When the search engine receives an alert, it denies further queries form detected victims 


\section{Containment of the Search Worm}

$\square \quad$ Is such a strategy possible?

$D_{t+1}=D_{t}+\left(I_{t}-D_{t}\right)\left[1-\left(1-\mu^{\delta}\right]\right.$ nodes by the time $t+1$

Probability that an infected sever scans a honey page during an infection cycle

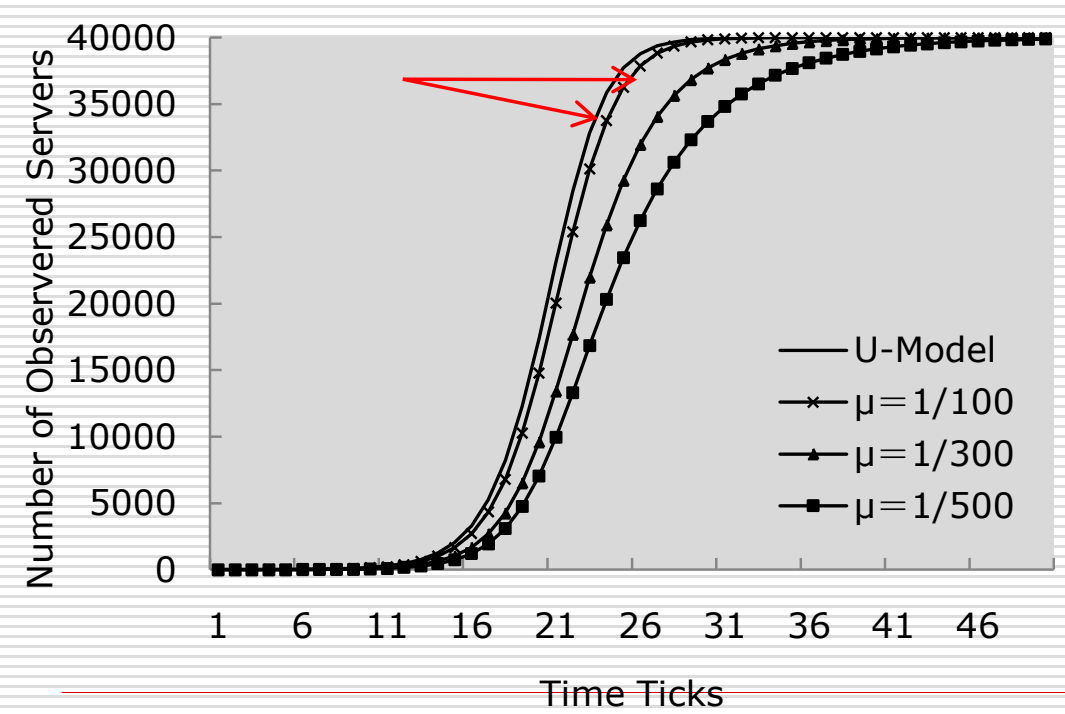

An infected sever can be induced to scan honeypots in a very short time after it is infected even if the insert rate is very small. 


\section{Containment of the Search Worm}

$\square$ Two questions:

- Containment requirement $\rightarrow$ Insert Rate?

$$
\mu=1-\left[1+\frac{\gamma \varepsilon \delta}{\ln (1-\gamma)}\right]^{1 / \delta} \quad \begin{aligned}
& \gamma \text { is the final prevalence rate: } \\
& \text { ration of infected vulnerable } \\
& \text { servers }
\end{aligned}
$$

- Is arbitrary requirement can be reached?

$\varepsilon \delta \leq 1$ No limitation, arbitrary requirement can be reached

$\varepsilon=\frac{V}{N}$ is the density of vulnerable servers. 


\section{Containment of the Search Worm}

$\square$ Effectiveness for the Santy worm

$\mathrm{N}=6,000,000 \quad \mathrm{~V}=40,000$

To contain the final prevalence rate below $1 \%$

\section{Insert rate $\rightarrow 0.011$}

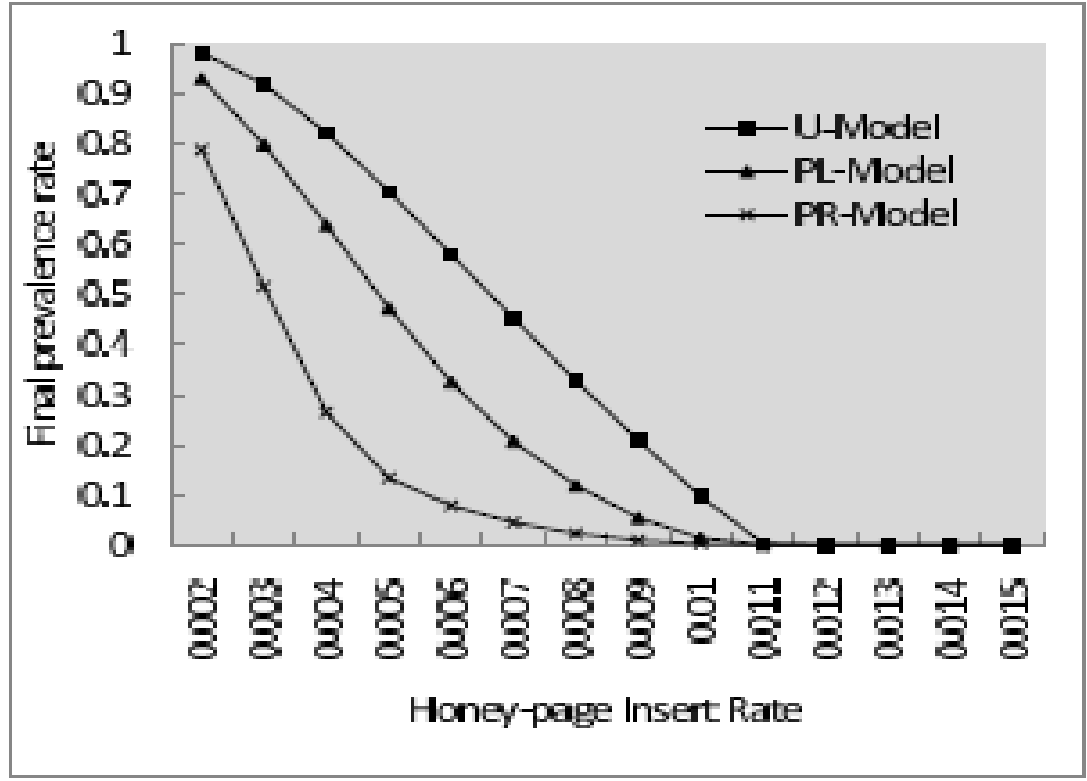

2 honey pages in every 100 search results can stop the spreading of the Santy worm at its early age! 


\section{Conclusion}

$\square$ Modeling of the Search Worm

- Eigenpages Distribution: Uniform distribution optimizes the spreading

- Page Ranking: slow downs the spreading

$\square$ Containment of the Search Worm

- Honey page insertion

- A small insert rate can lead a good containment effect 


\section{Challenging Future Work}

$\square$ Worm may validate the truth of the search results. Then, how to disguise honey pages as true ones both in URL and contents?

$\square$ Our current conclusions are based on simulation. Real experiments are required to verify them. 


\section{Question \& Answer}

\section{Thanks for your attention!}

